Research Paper

\title{
Forsythoside A exerts antipyretic effect on yeast-induced pyrexia mice via inhibiting transient receptor potential vanilloid 1 function
}

\author{
Cuiling Liu*, Hongchang Su*, Hongye Wan, Qingxia Qin, Xuan Wu, Xiangying Kong ${ }^{\bowtie}, \mathrm{Na}$ Lin ${ }^{\bowtie}$ \\ Institute of Chinese Materia Medica, China Academy of Chinese Medical Sciences, Beijing, China. \\ * These authors as first authors contributed equally to this article. \\ $\square$ Corresponding authors: Institute of Chinese Materia Medica, China Academy of Chinese Medical Sciences, No. 16, Nanxiaojie, Dongzhimennei, Beijing \\ 100700, China. Tel: +861064014411-2869 Fax: +861064013996. E-mail: xykong@icmm.ac.cn, nlin@icmm.ac.cn. \\ (C) Ivyspring International Publisher. This is an open access article distributed under the terms of the Creative Commons Attribution (CC BY-NC) license \\ (https://creativecommons.org/licenses/by-nc/4.0/). See http://ivyspring.com/terms for full terms and conditions.
}

Received: 2016.10.23; Accepted: 2016.11.12; Published: 2017.01.01

\begin{abstract}
Transient receptor potential vanilloid 1 (TRPV1) is a non-selective cation channel gated by noxious heat, playing major roles in thermoregulation. Forsythoside A (FT-A) is the most abundant phenylethanoid glycosides in Fructus Forsythiae, which has been prescribed as a medicinal herb for treating fever in China for a long history. However, how FT-A affects pyrexia and what is the underlying molecular mechanism remain largely unknown. Here we found that FT-A exerted apparent antipyretic effect through decreasing the levels of prostaglandin $\mathrm{E}_{2}\left(\mathrm{PGE}_{2}\right)$ and interleukin 8 (IL-8) in a dose-dependent fashion on the yeast induced pyrexia mice. Interestingly, FT-A significantly downregulated TRPVI expression in the hypothalamus and dorsal root ganglion

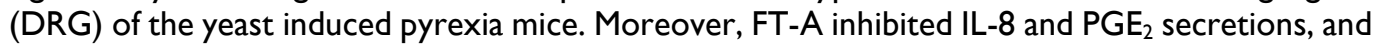
calcium influx in the HEK 293T-TRPV1 cells after stimulated with capsaicin, the specific TRPV1 agonist. Further investigation of the molecular mechanisms revealed that FT-A treatment rapidly inhibited phosphorylation of extracellular signal-regulated kinase (ERK), Jun $\mathrm{N}$-terminal kinase (JNK) and p38 in both yeast induced pyrexia mice and HEK 293T-TRPVI cells. These results suggest that FT-A may serve as a potential antipyretic agent and the therapeutic action of Fructus Forsythiae on pyretic related disease is, in part, due to the FT-A activities.
\end{abstract}

Key words: Transient receptor potential; Fever; Forsythoside A, Mitogen-activated protein kinases.

\section{Introduction}

Fever is a complex pathological process that manifests itself as a regulated elevation of body temperature over the normal circadian variations. Although fever may be regarded as a host defense response, it has potentially harmful consequences such as convulsions, dehydration, especially brain damage and seizures usually happen during severe and long-lasting fever in children [1]. Therefore, quite a number of people think that fever is a disease rather than a symptom or sign of illness and it is important to keep the body temperature in the state of homeostasis within the narrow range.

As a thermoregulatory manifestation to systemic inflammation, fever has been studied for years. Our understanding of the molecular mechanisms has substantially advanced over the past decade, and major advances have been made since the discovery of the transient receptor potential (TRP) family. They are strong candidates for a role in temperature sensing and regulating as evidenced by gating at different temperature thresholds [2]. For example, transient receptor potential vanilloid type 1 (TRPV1) is the sensor for uncomfortably warm $\left(>43^{\circ} \mathrm{C}\right)$ temperatures $[3,4]$, whereas TRPM8 and TRPA1 appear to function in our perception of cool temperatures $[5,6]$. There is an abundance of 
literature reporting that TRP channels may be activated by potential endogenous activators and regulators produced in inflammatory tissues [7]. Interestingly, these TRPs can also be activated by compounds from foods or traditional Chinese medicines (TCM). For example, TRPV1 is activated by ingredients of spicy foods such as hot pepper (capsaicin) [8] and ginger (gingerols and shogaols) [9, 10]. TRPM8 is activated by ingredients of peppermint (menthol) [11]. And TRPA1 is activated by ingredients of galic (allicin)[12] and cinnamon (cinnamaldehyde) [13]. Fructus Forsythiae, the fruit of Forsythia suspense (Thunb.) Vahl (Oleaceae), is a widely used traditional Chinese medicine (TCM) in the treatment of inflammation, pyrexia or emesis. Forsythoside A (FT-A) is one of the main phenylethanoid glycosides, and the most abundant active ingredients in Fructus Forsythiae, which possesses strong antimicrobial, antiviral and antioxidative activities [14, 15]. According to the literature and the clinical practice in TCM, Fructus Forsythiae shows obvious efficacy in alleviating fever. However, the antipyretic effect of FT-A was poorly understood.

In the present study, yeast induced pyrexia mice model was used to investigate the antipyretic effect of FT-A. Given the predominant function in thermoregulation of TRPs, particularly, we focused on whether FT-A has any influences on the TRPs and their downstream cell signaling in hypothalamus and dorsal root ganglion (DRG)of yeast-induced pyrexia mice. In addition, TRPV1-transfected HEK293T cells were used to investigate the effect of FT-A on capsaicin (CAP) induced calcium influx, IL-8 and $\mathrm{PGE}_{2}$ production.

\section{Material and Methods}

\section{Ethics statement}

The study was approved by the Research Ethics Committee of Institute of Chinese Materia Medica, China Academy of Chinese Medical Sciences, Beijing, China. All animals were treated in according with the guidelines and regulations for the use and care of animals of the Center for Laboratory Animal Care, China Academy of Chinese Medical Sciences.

\section{Chemicals and antibodies}

Forsythoside A (purity > 99\%) was purchased from the Chinese National Institute for the Control of Pharmaceutical and Biological Products (Beijing, China), which structural formula was shown in Figure 1. Antibodies used in the study included the following: Antibodies against TRPV1, TRPA1 and TRPM8 were obtained from Alomone labs (Alomone labs, Israel), Antibodies against p-extracellular signal-regulated kinase (ERK), p-Jun N-terminal kinase (JNK), p-P38 and GAPDH were supplied from Cell Signaling Technology (Beverly, MA, USA). The enhanced chemiluminescence (ECL) kit was from Bio-Rad; EDTA-free protease inhibitor was from Roche. Lipofectamine 2000 and Fluo 4-AM were acquired from Invitrogen (Carlsbad, CA, USA). Enzyme-linked immunosorbent assay (ELISA) kits for IL-8 and PGE $_{2}$ were purchased from R\&D Systems (Minneapolis, MN, USA). Other chemical agents were purchased from Sigma-Aldrich (St Louis, MO, USA).

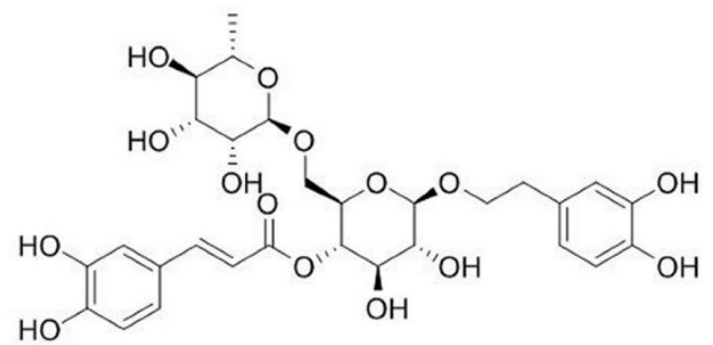

Figure 1. The chemical structure of Forsythoside A.

\section{Yeast induced pyrexia mice model}

Mice were housed in groups of four per cage, and maintained in a room with controlled temperature $\left(24 \pm 1^{\circ} \mathrm{C}\right)$, and maintained on a $12 \mathrm{~h}$ light/dark cycle and given ad libitum access to food and water. Pyrexia was induced in mice as described previous [16]. Briefly, Pyrexia was induced by injecting $20 \mathrm{mg} / \mathrm{kg}$ of Brewer's yeast suspension or vehicle (pyrogen-free $0.9 \% \mathrm{NaCl}$ ) subcutaneously in the back behind of the neck. The rectal temperature $\left(T_{R}\right)$ was recorded by an electronic thermometer into the rectum. The rise in $T_{R}$ was recorded after $17 \mathrm{~h}$. The induction of pyrexia was confirmed by a rise in temperature of more than $1^{\circ} \mathrm{C}$ and animals showing a rise in temperature less than $0.5^{\circ} \mathrm{C}$ were excluded from the experiment.

Forty pyretic animals were intraperitoneal injected with FT-A at doses of 2, 4 and $8 \mathrm{mg} / \mathrm{kg}$, and those who received Aspirin at a dose of $90 \mathrm{mg} / \mathrm{kg}$ were used as positive control. Those who administrated with saline were set as control.

\section{Cell culture and transfection}

Human embryonic kidney (HEK293T) cells were purchased from the National Institute of Cells (Beijing, China), and cultured in Dulbecco's modified Eagle's medium (DMEM; Invitrogen, USA) supplemented with $10 \%$ fetal bovine serum (FBS, Gibco, USA), penicillin $(100 \mathrm{U} / \mathrm{ml})$, and streptomycin $(100 \mu \mathrm{g} / \mathrm{ml})$. The cells were maintained at $37^{\circ} \mathrm{C}$ in a humidified chamber supplemented with $5 \% \mathrm{CO}_{2}$. Human TRPV1 expression vector (pcDNA3.0-TRPV1) was generously provided by Prof. Wan You, 
University of Peking. For transfection with TRPV1 plasmids, the HEK293T cells were cultured in a 6-well plate $\left(5 \times 10^{5}\right.$ cells/well $)$ overnight and transfected with pcDNA3.0-TRPV1 or control vector (pcDNA3.0) by lipofectamine 2000 (Invitrogen, USA) according to the manufacturer's instructions. These transfected cells were then subjected for further experiments.

\section{Intracellular calcium fluorescence imaging}

TRPV1-overexpressedHEK 293T cells were pretreated with FT-A for $2 \mathrm{~h}$. To measure calcium influx by confocal microscope, the dye Fluo-4 AM were loaded into HEK 293T-TRPV1 cells for 30min at $37^{\circ} \mathrm{C}$, then Pluronic F-127 for 30 min and Hanks' balanced salt solution $(\mathrm{PH}=7.4)$ were added in the system. Cells were then challenged with $8 \mu \mathrm{MCAP}$ in the dark. $\left[\mathrm{Ca}^{2+}\right]$ in measurements were carried out using an OLYMPUS FV1000 (Tokyo, Japan) confocal microscope at room temperature. Fluo-4AM was excited at $494 \mathrm{~nm}$, and the emission was recorded at $516 \mathrm{~nm}$. The measured fluorescence was subtracted from the background and auto fluorescence. The fluorescent intensity was used to represent the changes in intracellular $\mathrm{Ca}^{2+}$ levels. The experiments were carried out 3 times in triplicate measurements.

\section{Flow cytometry}

TRPV1-overexpressedHEK 293T cells were pretreated with a serial of concentration of FT-A (0.1, $1.0,10 \mu \mathrm{g} / \mathrm{mL}$ ) for $2 \mathrm{~h}$. Cells were digested and collected by centrifugation and loaded with Fluo-4/AM and incubated for $35 \mathrm{~min}$ at room temperature in normal physiological buffer (NPB) containing $1.0 \mathrm{mM} \mathrm{CaCl} 2$ and $0.1 \%(\mathrm{wt} / \mathrm{vol}$ ) BSA. The cells were washed twice and resuspended in NPB containing $1.0 \mathrm{mM} \mathrm{CaCl}_{2}$. Cells were then challenged with $8 \mu \mathrm{MCAP}$ in the dark. Fluorescence intensity was determined by flow cytometry using CELL Quest software with excitation at $488 \mathrm{~nm}$ and emission at $530 \mathrm{~nm}$. The experiments were carried out 3 times in triplicate measurements.

\section{Enzyme-linked immunosorbent assay}

Immediately after the last temperature measurements, blood was obtained from orbit, and then separated by centrifugation to obtain serum. The serum was aliquot and stored at $-80^{\circ} \mathrm{C}$.HEK 293T-TRPV1 cells were pretreated with FT-A for $2 \mathrm{~h}$ followed by stimulation with CAP $(8 \mu \mathrm{M})$ for $24 \mathrm{~h}$. Cell culture supernatants were collected. The levels of $\mathrm{PGE}_{2}$ and IL-8 in the cell culture supernatants and serum of mice were detected by ELISA Kit (R\&D Systems, Minneapolis, USA) according to the manufacturer's instructions. The absorbance was read at the wavelength of $450 \mathrm{~nm}$ with a microplate reader
(Lab systems Dragon, Helsinki, Finland). The experiments were carried out 3 times in triplicate measurements.

\section{Immunohistochemical analysis}

Paraffin sections $(4 \mu \mathrm{m})$ of tissue from the hypothalamus and DRG were mounted on slides. The sections were dewaxed by routine method and incubated with $3 \% \mathrm{H}_{2} \mathrm{O}_{2}$ for 10 min, and blocked by corresponded serum for $1 \mathrm{~h}$ at room temperature. Then the sections were incubated with different primary antibodies: p-ERK (1:100, Cell Signaling Technology, USA), p-JNK (1:100, Cell Signaling Technology, USA) and p-P38(1:100, Cell Signaling Technology, USA) at $4^{\circ} \mathrm{C}$ overnight, respectively. For the control staining, PBS was used instead of the primary antibody. Then incubated with second antibody and stained with 3, 3-diaminobenzidine (DAB). In this procedure, rinsing with PBS was carried out between any two steps. Image-Pro Plus 6.0 System (IPP) image analysis system was used for quantitative analysis. Five fields of view were randomly selected from each slice, and an index of positive staining was determined from the area of positive staining and the optical density. The positive index was calculated as positive area integral optical density (IOD).

\section{Western blotting analysis}

Immediately after the last temperature measurements, the mice were sacrificed by rapid decapitation. The hypothalamus and the DRG were rapidly removed, frozen in liquid nitrogen and placed in $-80^{\circ} \mathrm{C}$. Total protein of hypothalamus and the DRG were extracted following the instructions of the Bradford Kit. For in vitro experiments, HEK 293T-TRPV1 cells were pretreated with FT-A for $2 \mathrm{~h}$ followed by stimulation with CAP $(8 \mu \mathrm{M})$ for $15 \mathrm{~min}$. Cells was collected and total proteins were extracted. For immunoblot analysis, equivalent amounts of protein were separated in $8 \%$ SDS-PAGE and transferred to polyvinylidene fluoride membrane (Millipore, Massachusetts, USA), which was blocked by $5 \%$ nonfat dry milk. The membrane was then incubated with appropriately diluted primary antibodies overnight at $4^{\circ} \mathrm{C}$, followed by horseradish peroxidase-conjugated secondary antibodies for $1 \mathrm{~h}$ at room temperature (RT). The immune reactive bands were detected by ECL (Bio-Rad) and visualized with the Fusion-Capt Advance (VILBER, France). The intensity of the blots was quantified by MultiGauge software according to the manufacturer's instruction.

\section{Statistical Analysis}

Each experiment was repeated at least three times. Data were represented in the form of means \pm 
SD. All the data were analyzed using the SPSS statistical package (version 13.0, USA). The data were analyzed by One-way ANOVA followed by Dunnett's t-test to assess the statistical significance of the differences between the study groups. Differences were considered statistically significant when $P$ was less than 0.05 .

\section{Results}

\section{FT-A significantly prevents yeast-induced pyrexia and inhibits production of pyrogenic factors}

We first investigate the effects of FT-A on body temperature using yeast-induced pyrexia mice model. As shown in Figure 2A, subcutaneous (s.c.) injection of yeast evoked a significant elevation of rectal temperature $\left(T_{R}\right) 16 \mathrm{~h}$ later, and reached the peak within $18 \mathrm{~h} \quad\left(39.28 \pm 0.41^{\circ} \mathrm{C}\right.$ versus $36.78 \pm 0.36^{\circ} \mathrm{C}$ for vehicle, $P<0.01)$, then declined gradually during time period observed. However, the elevation of $T_{R}$ was significantly suppressed by FT-A at the doses of 2, 4 and $8 \mathrm{mg} / \mathrm{kg}$ from the onset of the pyrexia for up to $6 \mathrm{~h}$ after treatment with the dose-dependent manner. Especially FT-A at $8 \mathrm{mg} / \mathrm{kg}$ exerted almost equivalent ability with aspirin $(90 \mathrm{mg} / \mathrm{kg})$. The overall changes of $T_{R}$ with time was also evaluated as area under the curve (AUC) and presented with column diagram. The results showed that yeast could cause significant increase of AUC, while FT-A could markedly decrease the AUC with the dose-dependent tendency (Figure 2B).

Pro-inflammatory mediators and cytokines play the critical intermediary role in the process of fever, of which $\mathrm{PGE}_{2}$ and IL-8 are of great importance. Data of the present experiments showed that mice challenged with yeast dramatically enhanced the productions of $\mathrm{PGE}_{2}$ and IL-8. FT-A $(4,8 \mathrm{mg} / \mathrm{kg})$ treatment produced a marked decrease in the levels of $\mathrm{PGE}_{2}$ and IL-8, but a relatively weaker reduction in the FT-A $(2 \mathrm{mg} / \mathrm{kg}$, Figure $2 \mathrm{C}$ and $\mathrm{D}$ ). These findings demonstrated that FT-A exert antipyretic effect on yeast-induced fever by inhibiting the production of pyrogenic cytokines.

\section{A}

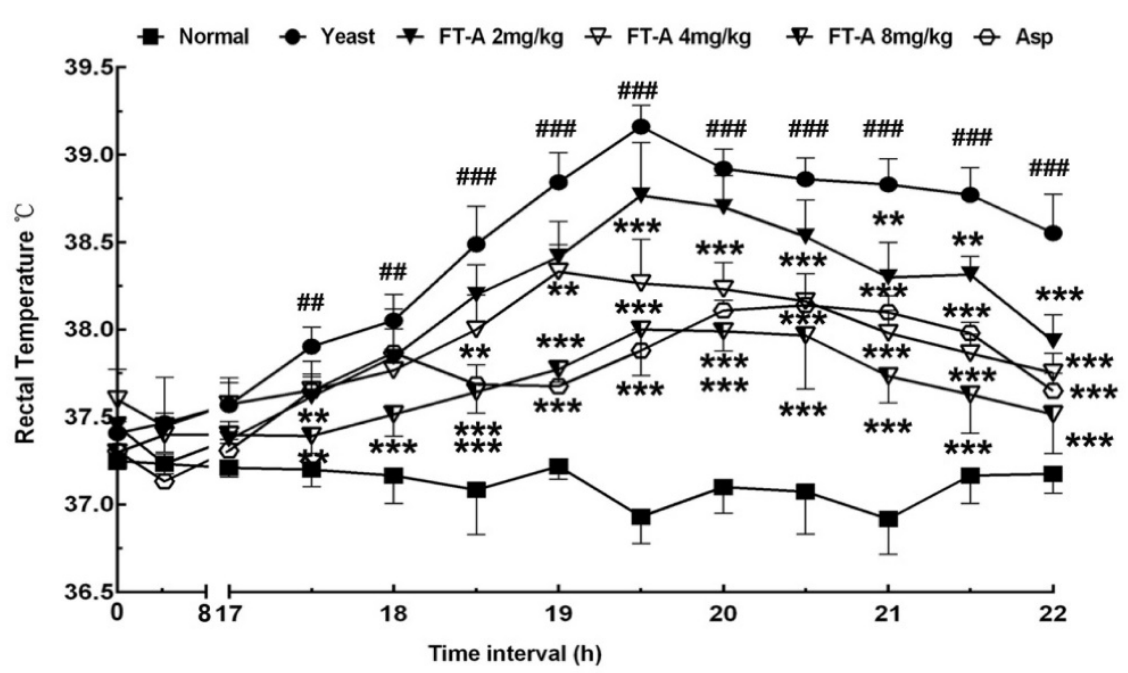

B

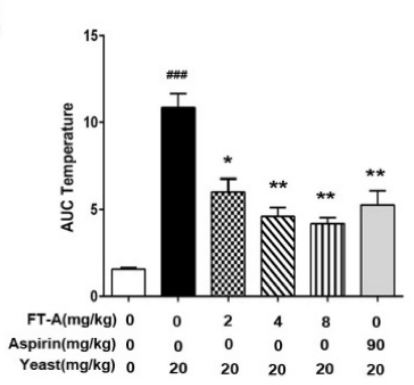

C

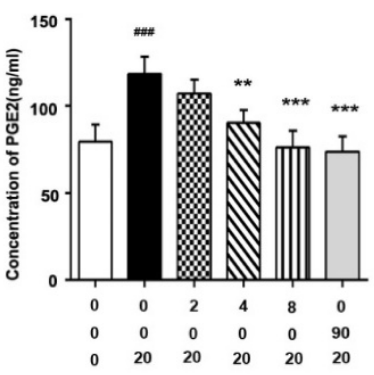

D

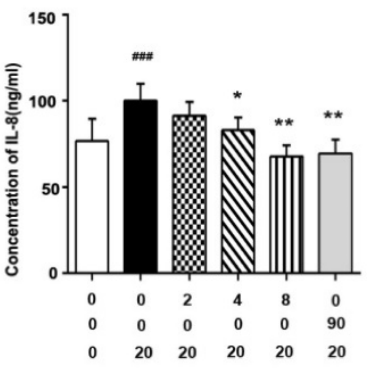

Figure 2. FT-A attenuates yeast-induced increase of rectal temperature and pyrogenic cytokines in the plasma. (A)Intraperitoneal injection of FT-A significantly antagonized yeast-induced pyrexia on mice. (B) Changes of rectal temperature expressed by area under the curve after yeast-induced pyrexia ( $t=17$ to 22 ). (C) $P G E_{2}$ and IL-8 levels in the

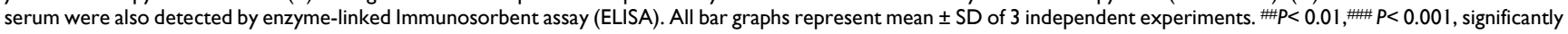
different from the Normal group; $* P<0.05$, $* * P<0.01$, $* * * P<0.001$, significantly different from the Yeast group. 


\section{FT-A markedly inhibits TRPVI expression in the hypothalamus and DRG of yeast-induced hyperthermia mice}

Since TRPs play an important role in the thermoregulation, next we investigated whether the anti-pyretic effects of FT-A related to TRPs. The hypothalamus has long been known as a major thermo-regulatory region of the CNS, and DRG neurons involved in almost all the thermal afferent pathways, so we detected the expression of TRPs subtypes in the two tissues of yeast-induced hyperthermia mice. As shown in Figure 3A, the expression of TRPV1 in hypothalamus was significantly up-regulated compared with saline injected control mice. Treatment of FT-A $(2,4,8$ $\mathrm{mg} / \mathrm{kg}$ ) significantly decreased the expression of TRPV1 in a dose-dependent fashion, with the most apparent decrease about $80 \%$ compared to the yeast injection mice. In contrast, the expressions of TRPA1 and TRPM8 in hypothalamus of yeast injection mice

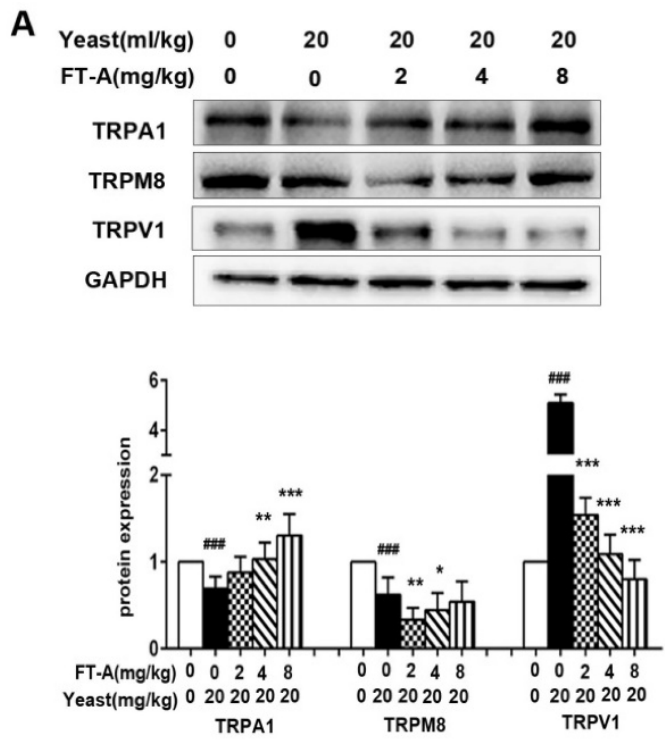

were markedly downregulated compared with saline injected control mice. FT-A ( 4 and $8 \mathrm{mg} / \mathrm{kg}$ ) increased TRPA1 protein expression $(P<0.05$ or $P<0.01)$. However, FT-A (4 and $8 \mathrm{mg} / \mathrm{kg}$ ) showed no obvious influence on TRPM8 expression, while FT-A at $2 \mathrm{mg} / \mathrm{kg}$ slightly inhibited TRPM8 expression.

Consistent with these findings, we also observed significantly increased TRPV1 expression in the DRG from yeast-induced hyperthermia mice (Figure 3B). FT-A at the concentration of $2 \mathrm{mg} / \mathrm{kg}$ showed no obvious effect on expression of TRPV1, while 4 and 8 $\mathrm{mg} / \mathrm{kg}$ of FT-A significantly inhibited the TRPV1 expression near the saline control level. The expressions of TRPA1 and TRPM8 in DRG were significantly decreased by yeast compared with saline injected control mice, while only $8 \mathrm{mg} / \mathrm{kg}$ of FT-A moderately increased the two-protein expression. Thus it can be seen that FT-A impacted TRPV1 expression most apparently.

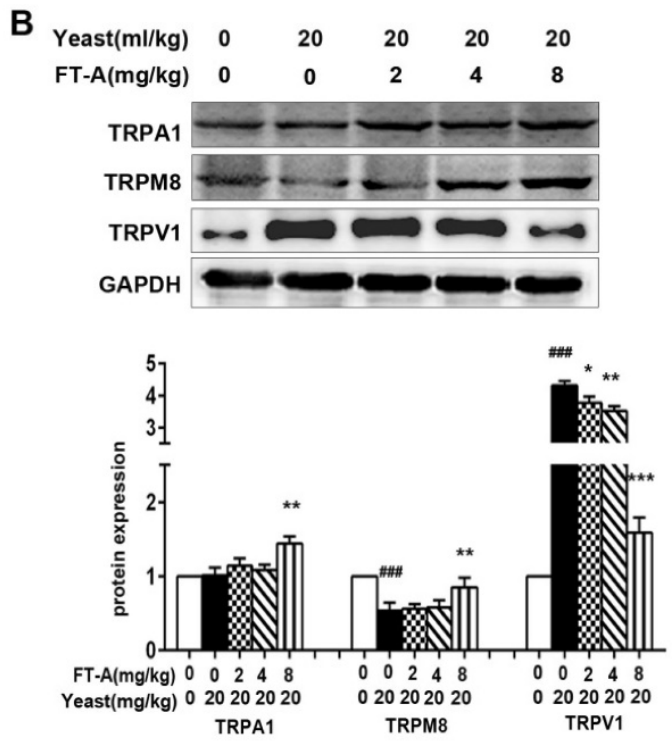

C
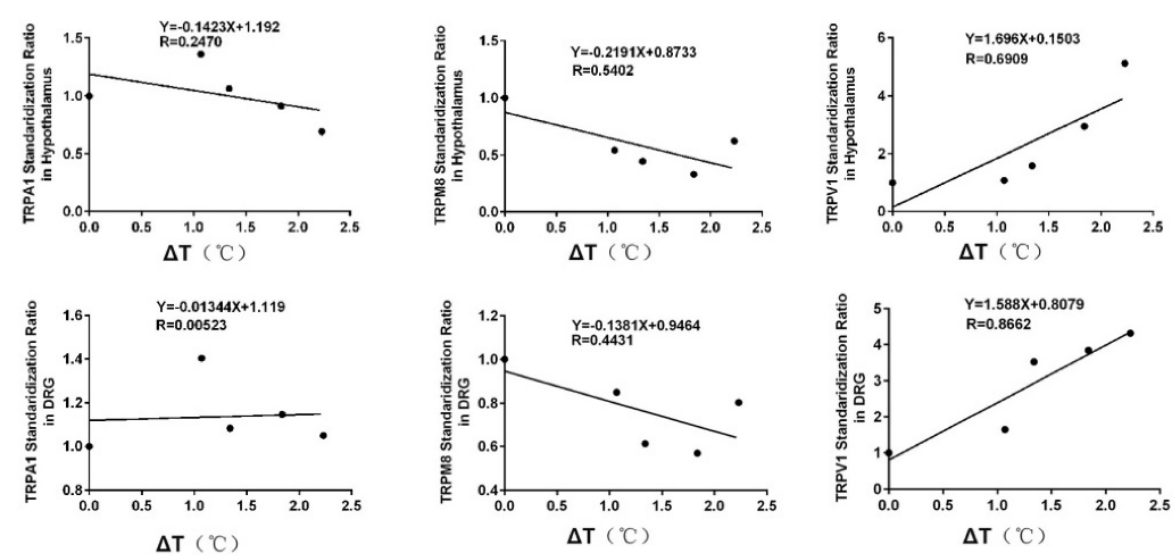

Figure 3. FT-Ainhibits TRPV1 expression while increases TRPAI and TRPM 8 expression in the hypothalamus and DRG of the mice with yeast-induced pyrexia. Expressions ofTRPV1, TRPA1 and TRPM8 were evaluated in the hypothalamus (A) and DRG (B) by Western blotting. Values are Mean \pm SD of 5 mice per group. \#P<0.05, \#P< 0.01 , \#\#P< 0.001 significantly different from the Normal group, $* P<0.05$, $* * P<0.01$, $* * * P<0.001$ significantly different from the Yeast group. (C)Correlations between the expression of TRPs and the change of rectal temperature were assessed in the mice with yeast-induced pyrexia ( $n=5$ samples per group). 
We therefore examined whether the expression levels of TRPV1, TRPA1 and TRPM8 can be correlated with the body temperature in yeast-induced fever. We identified a strong positive correlation between the expression of TRPV1 and body temperature. In contrast, the expressions of TRPA1 and TRPM8 showed moderately negative correlation with body temperature (Figure 3C). Together, these results demonstrate a pivotal role of TRPV1 in yeast-induced hyperthermia as well as in the antipyretic effect of FT-A.

\section{FT-A inhibitsIL-8 and PGE $_{2}$ secretions and calcium influx inTRPVI expressing cells}

To test the effect of FT-A on the TRPV1 activation, we transiently transfected HEK 293T cells with TRPV1 constructs. As expected, a considerable amount of TRPV1 was detected in HEK 293T-TRPV1 cells by western blot (Figure $4 \mathrm{~A}$ ). In this study, we also determined the levels of IL- 8 and $\mathrm{PGE}_{2}$ in the culture supernatants by ELISA.As shown in Figure 4B and $\mathrm{C}$, the levels of $\mathrm{PGE}_{2}$ and IL-8 released from HEK 293T cells were significantly increased after stimulated with $8 \mu \mathrm{M}$ CAP, the specific TRPV1 agonist $(P<0.05)$. When pretreatment with various concentrations of FT-A, the production of $\mathrm{PGE}_{2}$ and IL-8 induced by CAP were reduced in a dose-dependent manner. These data suggested that the inhibitory role of FT-Aon the releases of IL-8 and $\mathrm{PGE}_{2}$ inducted by CAP was mediated by TRPV1.

Stimulation of TRPV1 by CAP increases $\mathrm{Ca}^{2+}$ influx in heterologous systems $[17,18]$, in the present experiment, the intracellular $\mathrm{Ca}^{2+}$ influx response to CAP were monitored in TRPV1-overexpressed HEK 293T cells loaded with Fluo-4 AM by confocal microscope. As showed in Figure 5A and B, CAP significantly increased the intracellular level of $\mathrm{Ca}^{2+}$ in TRPV1-overexpressed HEK293T cells, pretreatment with FT-A $(0.1,1,10 \mu \mathrm{g} / \mathrm{mL})$ markedly abrogated the CAP-induced increase in intracellular $\mathrm{Ca}^{2+}$ level with a dose-dependent fashion.

To corroborate the finding that FT-A suppressed TRPV1 function, flow cytometry was performed to detect the intracellular $\mathrm{Ca}^{2+}$ level. Consistent with the confocal results, CAP induced $\mathrm{Ca}^{2+}$ influx in HEK 293T-TRPV1 cells up to $212.6 \%$ of the control. However, FT-A $(0.1,1,10 \mu \mathrm{g} / \mathrm{mL})$ significantly decreased CAP induced $\mathrm{Ca}^{2+}$ influx in HEK 293T-TRPV1 cells by $18.32 \%(P<0.05), 26.93 \%(P<0.01)$ and $39.15 \%(P<0.01)$, respectively (Figure $5 \mathrm{C}$ and $\mathrm{D})$. These results suggested that FT-A treatment inhibited the function of TRPV1 by suppressing TRPV1 sensitivity.

\section{FT-A suppresses the MAPKs pathway activation in the yeast-induced hyperthermia mice and TRPVI expressing cells}

Previous studies indicate that a global activation of MAPK signaling occurs during the process of hyperthermia $[19,20]$. To further investigate the role of FT-A in pyrexia, we examined if FT-A was able to inhibit certain signaling pathways associated with pyrexia. In this experiments, HEK 293T-TRPV1 cells were treated with FT-A, then stimulated with CAP, followed by analyzing the activation of the MAPK signaling pathway. As shown in Figure 6, CAP $(8 \mu \mathrm{M})$ exposure resulted in an increase of the phosphorylated form of ERK and p38 MAPK, but not JNK/SAPK MAPK branch. Interestingly, when we treated the cells with various doses of FT-A for $2 \mathrm{~h}$

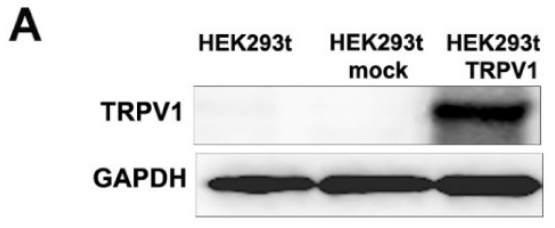

B

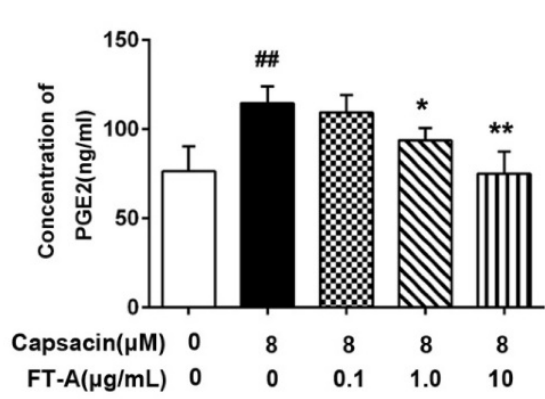

C

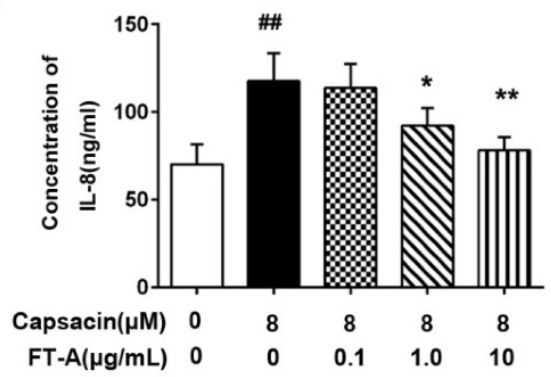

Figure 4. FT-A inhibits PGE 2 and IL-8 productions through TRPV1 signal. (A) Expression of TRPV1 protein in HEK 293T cells, HEK 293T mock cells and HEK 293T-TRPV1 cells by Western blot utilizing human anti-TRPV1 polyclonal antibody. HEK 293T-TRPV1 cells were incubated with $0.1,1$ and $10 \mu g / \mathrm{mL} \mathrm{FT-A} \mathrm{for} 2 \mathrm{~h}$, and then further treated with $10 \mu \mathrm{M}$ CAP for $24 \mathrm{~h}$. The levels of PGE $\mathrm{PE}_{2}(\mathrm{~B})$ and IL-8 $(\mathrm{C})$ in cell supernatants were detected by ELISA. Results were shown as mean \pm SD values of three independent experiments. \#P< 0.01, significantly different from the Control group; $* P<0.05, * * P<0.01$, significantly different from the CAP group. 
before stimulation with $\mathrm{CAP}$ and found that the activations of these signaling pathways were markedly inhibited in a dose dependent manner. Especially at the concentration of 1 and $10 \mu \mathrm{g} / \mathrm{mL}$ of FT-A induced a sharp decrease in their activity of ERK and $\mathrm{p} 38$.

To further investigate the role of FT-A on TRPV1, we detected the activation of MAPK signaling pathway on the hypothalamus and DRG of the yeast-induced fever mice. Immunohistochemical staining results showed that there was slight positive staining of the phosphorylated form of ERK, JNK and p38MAPK in the hypothalamus of the normal mice, while the signals significantly increased in the yeast-treated mice. However, the positive signals were remarkably reduced in the FT-A groups compared with that of the yeast group. Quantification of results showed that, compared to yeast group, there were obvious statistical significance in the FT-A-treated group (Figure $7 \mathrm{~A}$ and B, Figure $8 \mathrm{~A}$ and B). Downregulation of phosphorylation of all the three subtypes of MAPK was confirmed using Western blot in the hypothalamus and DRG of the yeast-induced fever mice (Figure $7 \mathrm{C}$ and Figure 8C). As to the difference of FT-A on JNK phosphorylation between HEK 293T-TRPV1 cells and the yeast-induced fever mice, we think that the HEK 293T-TRPV1 cells could not fully mimic the in vivo biological process, which is the universal limitation of the in vitro cell model. Taken together, the results demonstrate that FT-A suppressed MAPK activation in mediating downstream responses to TRPV1 activation.
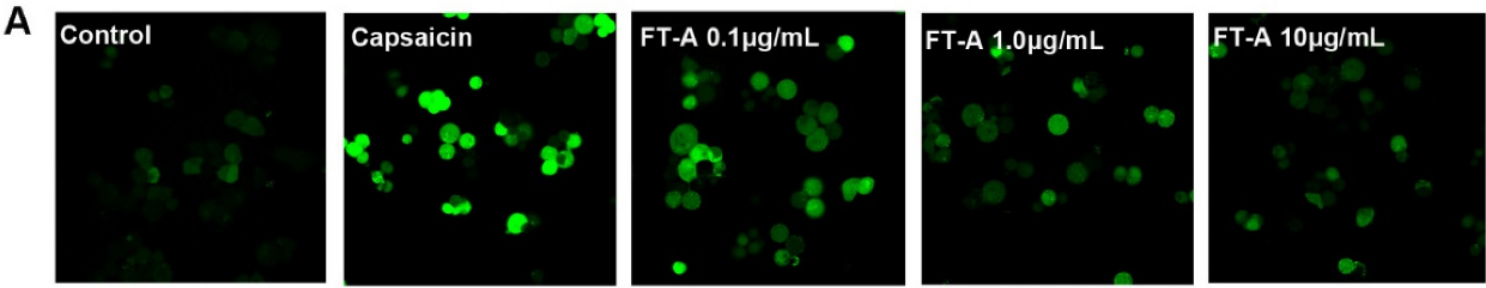

B

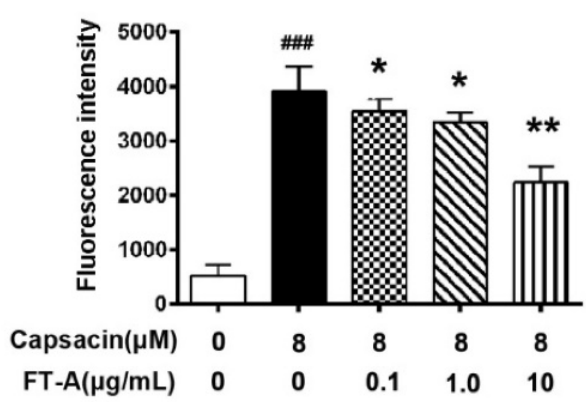

C

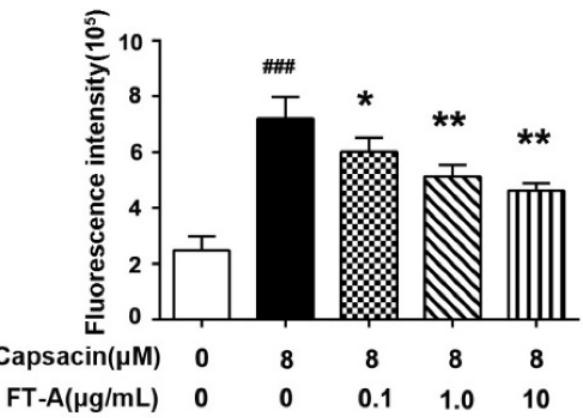

D
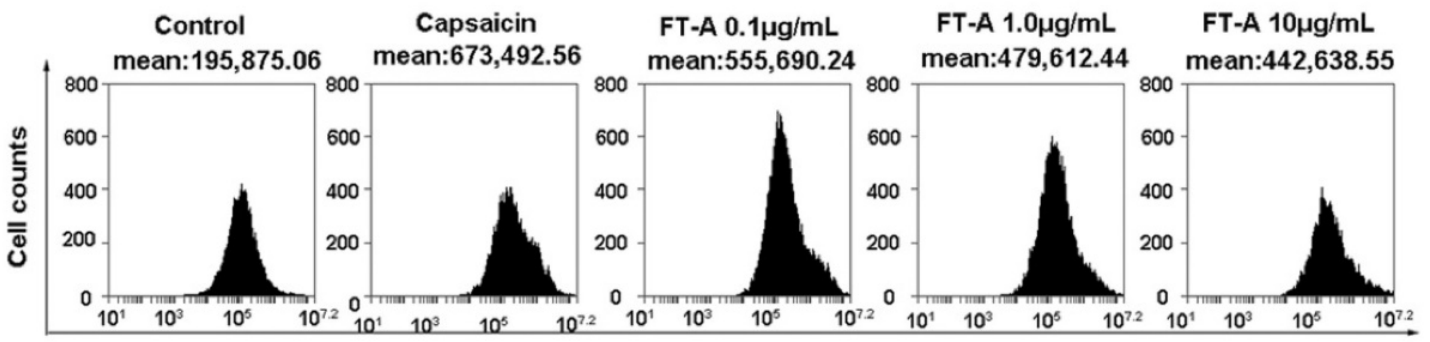

Fluorescence intensity

Figure 5. FT-A suppresses capsaicin-induced $\mathrm{Ca}^{2+}$ influx in HEK 293T-TRPV1 cells. (A). HEK 293T-TRPV1 cells were pre-incubated with $10 \mu M$ Fluo-4/AM fluorescence for 15 min at $37^{\circ} \mathrm{C}$, and then treated with $0.1,1$ and $10 \mu \mathrm{g} / \mathrm{Ml} \mathrm{FT-A}$ for $2 \mathrm{~h}$, with or without $10 \mu \mathrm{M}$ CAP for 15 min. Ca ${ }^{2+}$ influx was determined by Confocal microscopic analysis $(\mathrm{A})$ and flow cytometry (C). Quantitative analysis of the mean $\mathrm{Ca}^{2+}$ concentration for confocal microscopic analysis (B) and flow cytometry (D). Values are Mean \pm SD from 3 independent experiments. ${ }^{\ldots} P<0.001$ significantly different from the Control group; $* P<0.05, * * P<0.01$ significantly different from the $C A P$ group. 


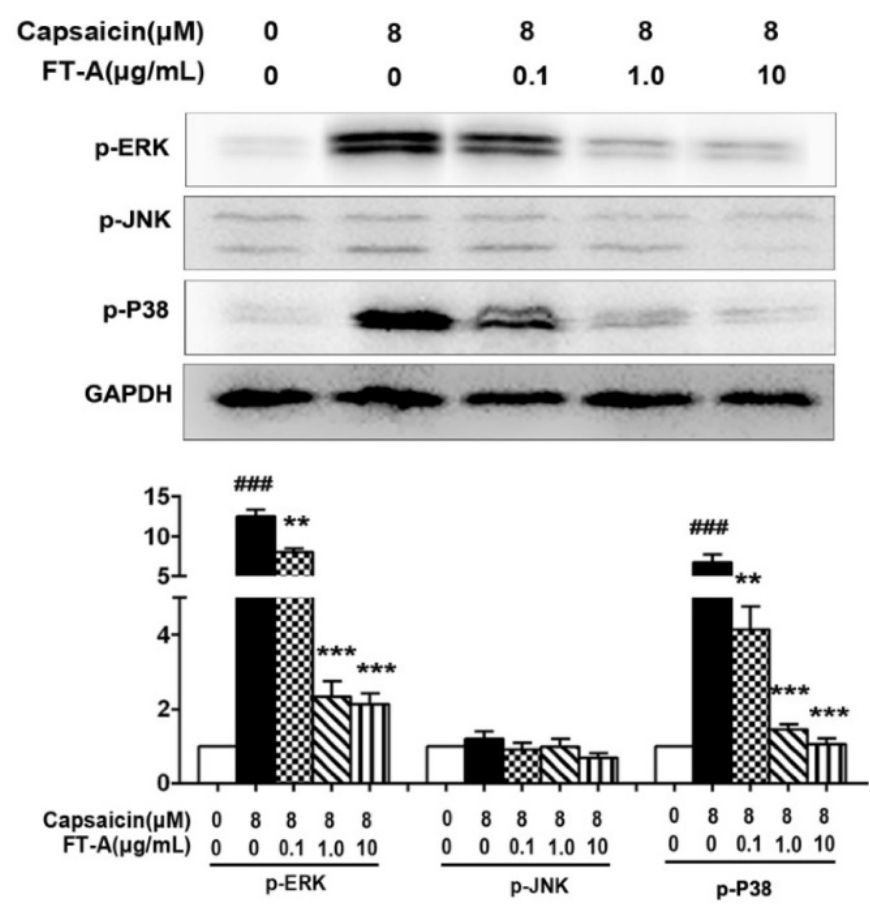

Figure 6. FT-A suppresses MAPKs activation in the HEK 293T-TRPV1 cells induced by CAP. (A) HEK 293T-TRPV1 cells were pre-incubated with or without of FT-A for $2 \mathrm{~h}$, following treatment with $10 \mu \mathrm{MCAP}$ for $15 \mathrm{~min}$. Total protein was extracted and subjected to Western blot analysis. And relative amounts of each protein were determined by densitometric analysis. One of three experiments with similar results is shown. Data are represented as the mean $\pm S D$. $P(0.001$ significantly different from the Control group; $* * P<0.01, * * * P<0.001$ significantly different from the CAP group.

\section{Discussion}

Fructus Forsythiae has been widely used in the treatment of various inflammatory febrile diseases in Chinese medical practice. Because of the beneficial clinical impact, it is necessary to understand how its active components exerts therapeutic function. FT-A, a phenylethanoid glycoside, the main and most abundant active ingredient in Fructus Forsythiae, has been known to possess antimicrobial and antiviral activities. However, its potential role in the antipyretic and its underlying pharmacological mechanism remain unclear. Results of the present study demonstrated that FT-A alleviated fever of yeast induced pyrexia mice via suppression TRPV1 expression and activation, inhibition MAPKs activation of the hypothalamus and DRG, and subsequently decreased secretion of pyretic cytokine as $\mathrm{PGE}_{2}$ and IL-8. Additionally, studies using the gene-manipulated strategy in TRP channel-deficient HEK293T cells further support the observation that FT-A suppressed TRPV1 activation through inhibiting MAPK pathway. The above findings were summarized in Figure 9. These findings provide the mechanistic explanation, at least in part for the antipyretic effect of FT-A against fever related disease, such as inflammatory fever.
Yeast induced pyrexia is called pathogenic fever by increasing the synthesis of prostaglandin, and is considered as a useful model for the screening of plants materials as well as synthetic drugs for their antipyretic effect [21, 22]. Several cytokines such as IL-8 and IL-1 are characterized as endogenous pyrogens, and the inhibition of these pyrogensis responsible for the antipyretic effect [21]. In the present study, intraperitoneal administration of FT-A significantly attenuated rectal temperature of yeast induced febrile mice. Therefore, it can be postulated that FT-A maybe interfere with the release of prostaglandin and pyrogenic cytokines. So, we tested the effect of FT-A on PGE 2 and IL-8 secretion by yeast induced pyretic animals. The data showed that FT-A reduced the $\mathrm{PGE}_{2}$ and IL-8 levels in febrile mice.

The molecular components and mechanisms involved in pyrexia formation are now starting to be unfolded. The identification and cloning of the TRPs, such as TRPV1, TRPM8 and TRPA1, represented a significant step in the clarification of the molecular mechanisms underlying thermoregulation of the body. Thermo sensitive TRPs were shown to be highly expressed in nociceptive pathways, specialized somato sensory neurons in the trigeminal and DRG that project axonal processes to the outer layers of the skin [23-25]. By contrast, expression levels for TRP channels in the brain are much lower than that in the DRG [26]. Notably, the channel activity of TRPV1 is remarkably up-regulated by inflammatory mediators [23]. Since TRPs receptor has emerged as a key therapeutic target for inflammatory fever management because of their central implication in thermoregulation, we observed whether the antipyretic effect of FT-A related to TRPs. Our present results revealed that FT-A could markedly inhibited protein expressions of TRPV1, and slightly increased the expressions of TRPA1 and TRPM8in the hypothalamus and DRG. Furthermore, we identified a strong positive correlation between the expression level of TRPV1 and the body temperature, while the expression levels of TRPA1 and TRPM8 were not obviously corrected with the body temperature, which implied that TRPV1 may contribute most to the anti-pyretic effect of FT-A.

Potentiating of TRPV1 channel activity, along with an incremented TRPV1 surface expression are major events underlying the nociceptors activation and sensitization that leads to thermal regulation [27]. At the molecular level, TRPV1 belongs to the TRP family of cation channels, and is a $\mathrm{Ca}^{2+}$-permeable, ionotropic receptor non-selective channel. Upon TRPV1activation by thermal stimuli or noxious chemical, leads to change of internal $\mathrm{Ca}^{2+}$, which itself represents a powerful influence on cellular signaling, 
feeding back upon TRPV1 function [28]. Increasing $\mathrm{Ca}^{2+}$ influx contributes to several pathological conditions, including pro-inflammatory cytokines secretion. In the present study, we constructed HEK 293T-TRPV1 cells, which stably overexpressed TRPV1, to explore the roles of TRPV1 on the anti-pyrexia effects of FT-A. Our results also showed that activation of TRPV1 by CAP resulted in marked increase of internal $\mathrm{Ca}^{2+}$, in turn, triggered the release of pro-inflammatory agents as IL-8 and $\mathrm{PGE}_{2}$, which were inhibited by FT-A in a concentration dependent manner. These results suggest that FT-A treatment inhibits the function of TRPV1 either by a decrease in the expression of TRPV1 or an inhibition in TRPV1 sensitivity.

Identified signal transduction events mediating the TRPV1 activation include transient intracellular
$\mathrm{Ca}^{2+}$ rises and phosphorylation of kinases belonging to the p38, ERK and JNK mitogen activated protein kinase (MAPK) cascades suggesting that all three MAPK pathways may be involved in downstream TRPV1 effects [29]. In the present study, we found that the activation of three subfamilies of MAPKs, including ERK, JNK, and p38 MAPKs in the DRG and hypothalamus of the pyrexia mice induced by yeast. However, the increased the activation of MAPKs was downregulated when administrated with FT-A, which indicated that ERK, JNK, and p38 MAPKs were involved in the antipyretic effect of FT-A. In support of this finding, immune reactivity assays demonstrated increased phosphorylation of MAPKs in hypothalamus and DRG of pyretic mice, while decreased in mice receiving FT-A treatment.

A

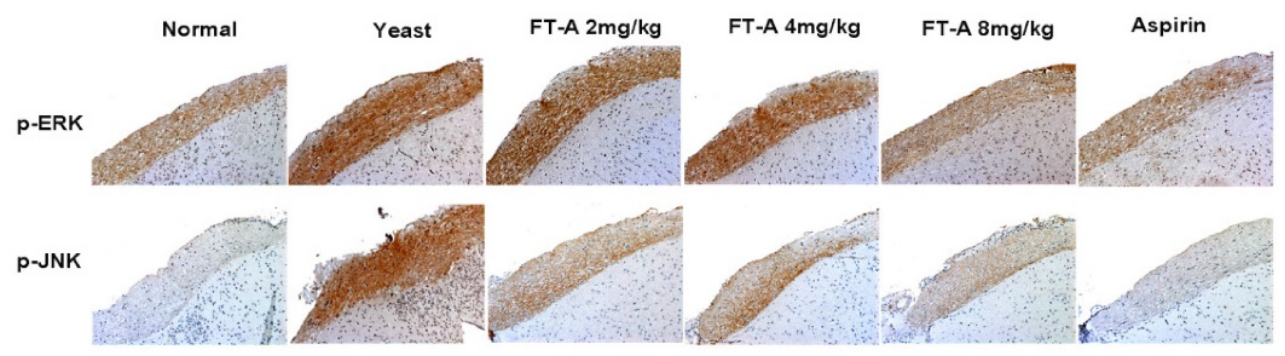

p-P38
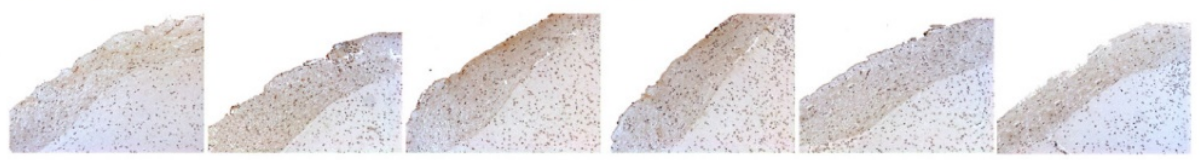

B
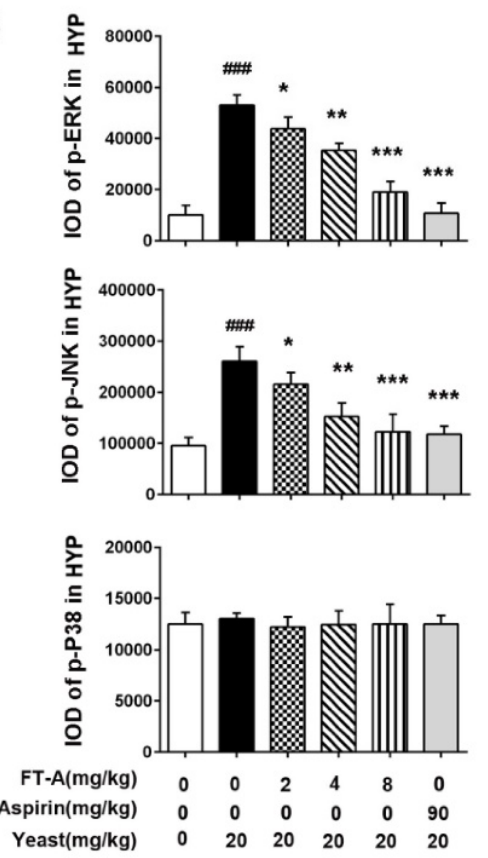
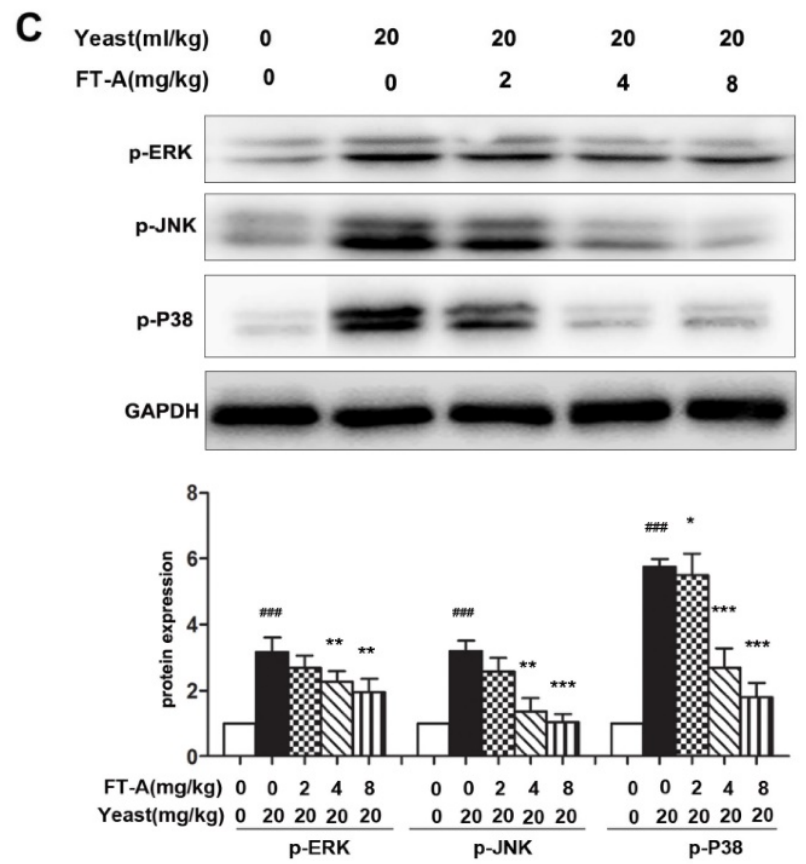

Figure 7. FT-A inhibits MAPKs activation in the hypothalamus on mice with yeast-induced pyrexia. The pyretic mice were intraperitoneal injected with FT-A at doses of 2,4 and $8 \mathrm{mg} / \mathrm{kg}$, and the expression of p-ERK, p-JNK and p-P38 in the hypothalamus were detected by immunohistochemical staining (A) and Western blotting (C). Quantitative analysis of the mean amount of each protein was determined by integral optical density. One of three experiments with similar results is shown. Data are represented as the mean \pm SD. \#m 0.001 , significantly different from the Normal group; $* P<0.05$, $* * P<0.01$, $* * * P<0.001$, significantly different from the Yeast group. 
A

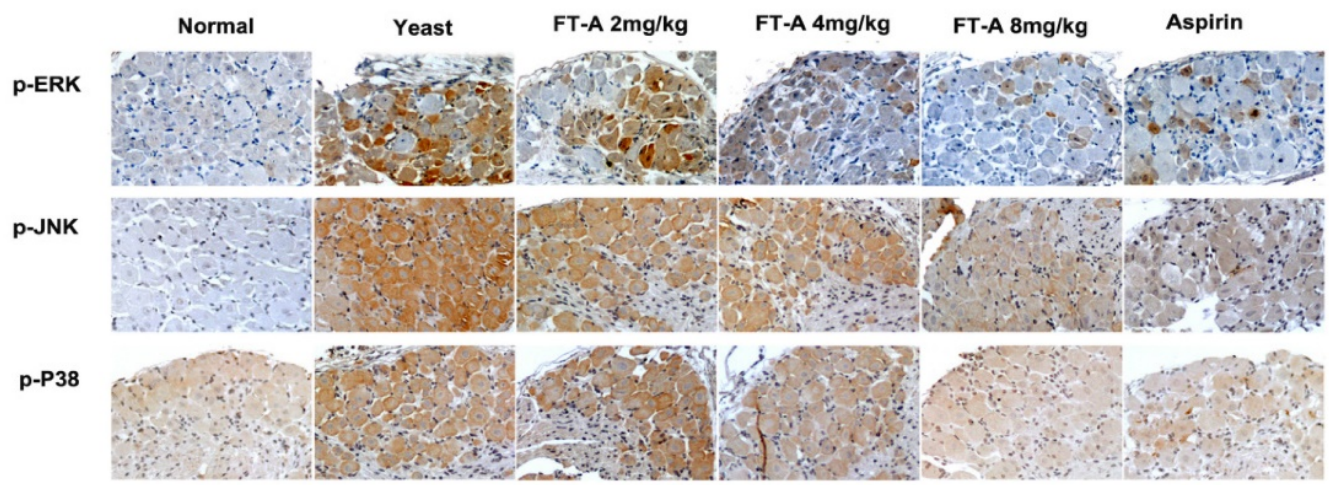

B
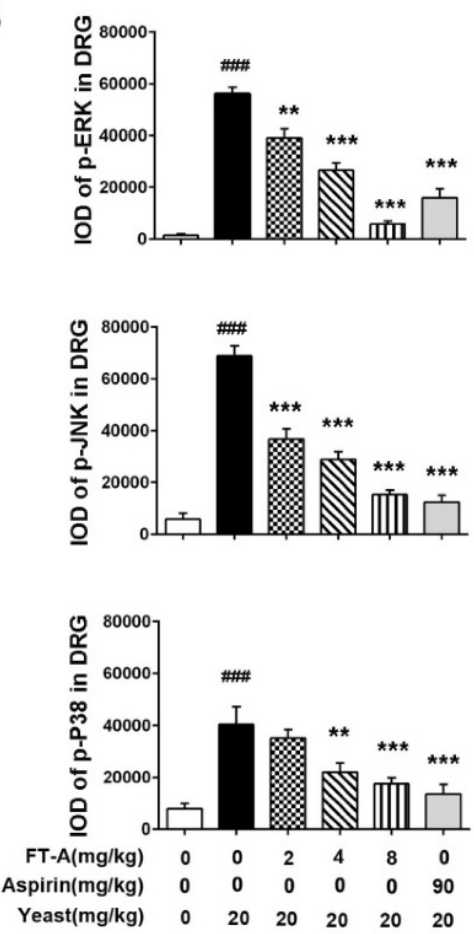

C
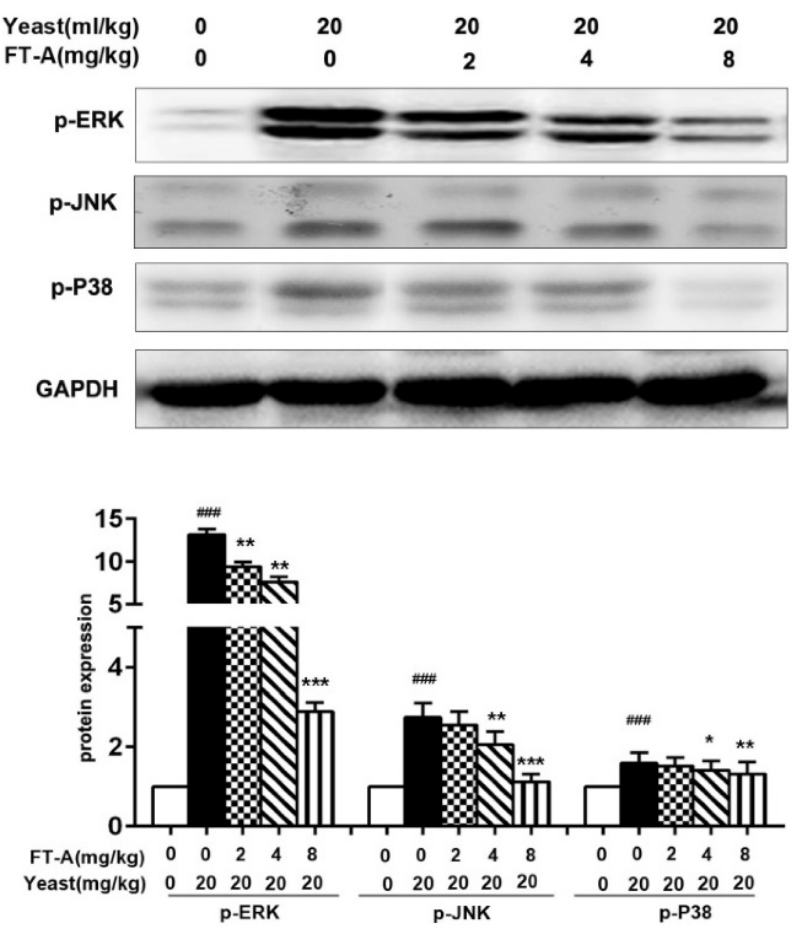

Figure 8. FT-A inhibits MAPKs activation in the DRG on mice with yeast-induced pyrexia. The pyretic mice were intraperitoneal injected with FT-A at doses of 2,4 and 8 $\mathrm{mg} / \mathrm{kg}$, and the expressions of p-ERK, p-JNK and p-P38 in the DRG were detected by immunohistochemical staining $(A)$ and Western blotting $(C)$. Quantitative analysis of the mean amount of each protein was determined by integral optical density. One of three experiments with similar results is shown. Data are represented as the mean \pm SD.\#P< $0.01 \ldots+\ldots<0.001$, significantly different from the Normal group; $* P<0.05$, $* * P<0.01$, **** $P<$ 0.001 , significantly different from the Yeast group.

Figure 9. Model depicting the proposed mechanism by which FT-A exert antipyretic effect. Target-derived pyrogenic cytokines such as $\mathrm{PGE}_{2}$ and IL-8, stimulated TRPVI activation, inducing $\mathrm{Ca}^{2+}$ influx, subsequently activated MAPKs signal pathway. At the nucleus, MAPKs signaling can increase TRPVI expression and pyrogenic cytokines. Consequently, the increased TRPVI could be transported to the membrane of the cell, and the elevated PGE 2 and IL-8 could be secreted out of the cell, and activated TRPVI. Red arrow mark indicates that an inhibition or a downregulation in this study observed.

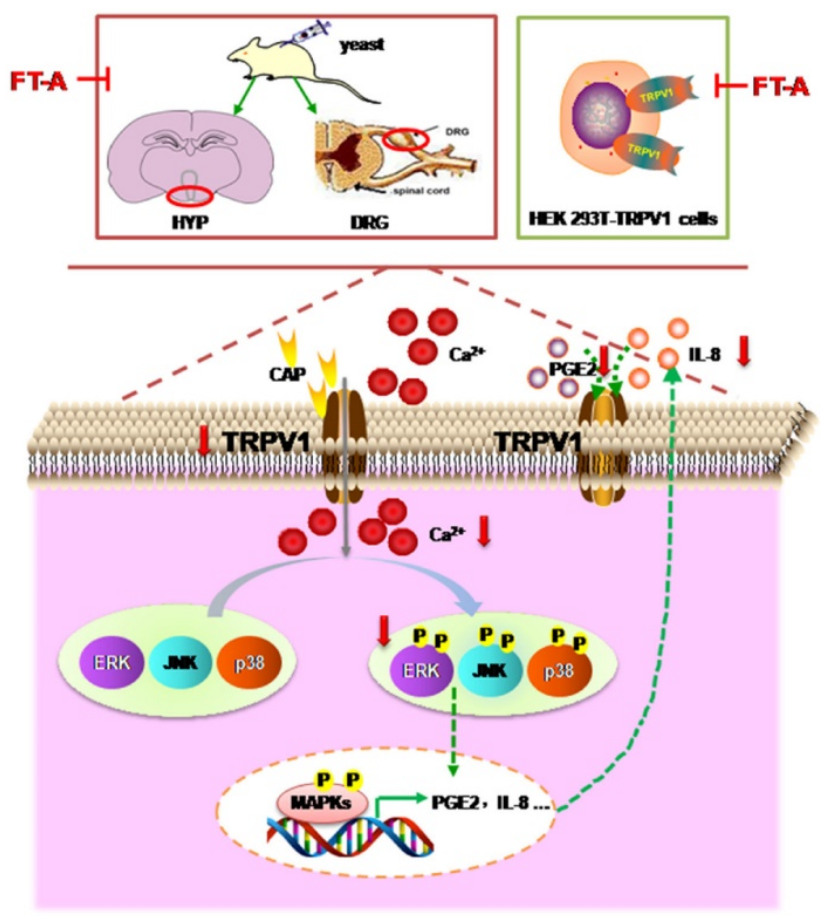


In summary, we have demonstrated that FT-A alleviates pyrexia through inhibiting the secretion of inflammatory mediators PGE $_{2}$ and IL-8, which may be associated with the suppression of the TRPV1 expression, decease of calcium influx, and inhibition of phosphorylation of MAPKs. Our findings provide new insights into the molecular mechanism underlying the effect of FT-A on pyrexia and suggest that FT-A may serve as a potent drug candidate for inflammatory fever, and that the therapeutic action of Fructus Forsythiae on pyretic related disease is, in part, due to the FT-A activities.

\section{Acknowledgments}

The investigation was supported by grants from the projects of China Academy of Chinese Medical Sciences (Z02096).

\section{Author contributions}

$\mathrm{N}$ Lin and $\mathrm{XY}$ Kong conceived and designed the experiments; CL Liu, HC Su, and HY Wan performed the experiments; $X \mathrm{Wu}$ and QX Qin analyzed the data; $X Y$ Kong wrote the manuscript and $N$ Lin reviewed it.

\section{Competing Interest} interests.

The authors declare no competing financial

\section{References}

1. Crocetti M, Moghbeli N, Serwint J. Fever phobia revisited: have parental misconceptions about fever changed in 20 years? Pediatrics. 2001; 107: 1241-6.

2. Patapoutian A, Peier AM, Story GM, Viswanath V. ThermoTRP channels and beyond: mechanisms of temperature sensation. Nature reviews Neuroscience. 2003; 4: 529-39.

3. Green D, Ruparel S, Gao X, et al. Central activation of TRPV1 and TRPA1 by novel endogenous agonists contributes to mechanical allodynia and thermal hyperalgesia after burn injury. Molecular pain. 2016; 12.

4. Wei NN, Lv HN, Wu Y, et al. Selective Activation of Nociceptor TRPV1 Channel and Reversal of Inflammatory Pain in Mice by a Novel Coumarin Derivative Muralatin L from Murraya alata. The Journal of biological chemistry. 2016; 291: 640-51.

5. Bandell M, Dubin AE, Petrus MJ, et al. High-throughput random mutagenesis screen reveals TRPM8 residues specifically required for activation by menthol. Nature neuroscience. 2006; 9: 493-500.

6. Kwan KY, Allchorne AJ, Vollrath MA, Christensen AP, Zhang DS, Woolf CJ, et al. TRPA1 contributes to cold, mechanical, and chemical nociception but is not essential for hair-cell transduction. Neuron. 2006; 50: 277-89.

7. Benemei S, Patacchini R, Trevisani M, et al. TRP channels. Current opinion in pharmacology. 2015; 22: 18-23.

8. Caterina MJ, Schumacher MA, Tominaga M, et al. The capsaicin receptor: a heat-activated ion channel in the pain pathway. Nature. 1997; 389: 816-24.

9. Morera E, De Petrocellis L, Morera L, et al. Synthesis and biological evaluation of [6]-gingerol analogues as transient receptor potential channel TRPV1 and TRPA1 modulators. Bioorganic \& medicinal chemistry letters. 2012; 22: 1674-7.

10. Watanabe T, Terada Y. Food Compounds Activating Thermosensitive TRP Channels in Asian Herbal and Medicinal Foods. Journal of nutritional science and vitaminology. 2015; 61 Suppl: S86-8.

11. Asuthkar S, Demirkhanyan L, Sun X, et al. The TRPM8 protein is a testosterone receptor: II. Functional evidence for an ionotropic effect of testosterone on TRPM8. The Journal of biological chemistry. 2015; 290: 2670-88.

12. Bautista DM, Movahed P, Hinman A, et al. Pungent products from garlic activate the sensory ion channel TRPA1. Proceedings of the National Academy of Sciences of the United States of America. 2005; 102: 12248-52.

13. Bandell M, Story GM, Hwang SW, et al. Noxious cold ion channel TRPA1 is activated by pungent compounds and bradykinin. Neuron. 2004; 41: 849-57.

14. $\mathrm{Ou} \mathrm{H}$, Zhang $\mathrm{Y}$, Wang $\mathrm{Y}$, et al. Antioxidant and antibacterial activity of two compounds (forsythiaside and forsythin) isolated from Forsythia suspensa. The Journal of pharmacy and pharmacology. 2008; 60: 261-6.
15. Wang Y, Zhao H, Lin C, et al. Forsythiaside A Exhibits Anti-inflammatory Effects in LPS-Stimulated BV2 Microglia Cells Through Activation of Nrf2/HO-1 Signaling Pathway. Neurochemical research. 2015.

16. Sengar N, Joshi A, Prasad SK, et al. Anti-inflammatory, analgesic and anti-pyretic activities of standardized root extract of Jasminum sambac. Journal of ethnopharmacology. 2015; 160: 140-8.

17. Garaud S, Taher TE, Debant M, et al. CD5 expression promotes IL-10 production through activation of the MAPK/Erk pathway and upregulation of TRPC1 channels in B lymphocytes. Cellular \& molecular immunology. 2016.

18. Sundivakkam PC, Natarajan V, Malik AB, et al. Store-operated Ca2+ entry (SOCE) induced by protease-activated receptor-1 mediates STIM1 protein phosphorylation to inhibit SOCE in endothelial cells through AMP-activated protein kinase and p38beta mitogen-activated protein kinase. The Journal of biological chemistry. 2013; 288: 17030-41.

19. Gourgou E, Aggeli IK, Beis I, et al. Hyperthermia-induced Hsp70 and MT20 transcriptional upregulation are mediated by p38-MAPK and JNKs in Mytilus galloprovincialis (Lamarck); a pro-survival response. The Journal of experimental biology. 2010; 213: 347-57.

20. Zhang T, Leng Z, Liu W, et al. Suppressed expression of mitogen-activated protein kinases in hyperthermia induced defective neural tube. Neuroscience letters. 2015; 594: 6-11.

21. Moltz H. Fever: causes and consequences. Neuroscience and biobehavioral reviews. 1993; 17: 237-69.

22. Jan S, Khan MR. Antipyretic, analgesic and anti-inflammatory effects of Kickxia ramosissima. Journal of ethnopharmacology. 2016; 182: 90-100.

23. Szolcsanyi J. Effect of capsaicin on thermoregulation: an update with new aspects. Temperature. 2015; 2: 277-96.

24. Ran C, Hoon MA, Chen X. The coding of cutaneous temperature in the spinal cord. Nature neuroscience. 2016; 19: 1201-9.

25. Caspani O, Zurborg S, Labuz D, et al. The contribution of TRPM8 and TRPA1 channels to cold allodynia and neuropathic pain. PloS one. 2009; 4: e7383.

26. Kauer JA, Gibson HE. Hot flash: TRPV channels in the brain. Trends in neurosciences. 2009; 32: 215-24.

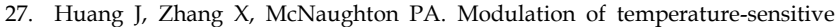
TRP channels. Seminars in cell \& developmental biology. 2006; 17: 638-45.

28. Straub RH. TRPV1, TRPA1, and TRPM8 channels in inflammation, energy redirection, and water retention: role in chronic inflammatory diseases with an evolutionary perspective. Journal of molecular medicine. 2014; 92: 925-37.

29. Chen Y, Geis C, Sommer C. Activation of TRPV1 contributes to morphine tolerance: involvement of the mitogen-activated protein kinase signaling pathway. The Journal of neuroscience : the official journal of the Society for Neuroscience. 2008; 28: 5836-45. 